\title{
Die Glaubwürdigkeit von Corporate Weblogs aus der Sicht der Blogger-Community
}

\author{
Teresa Naab / Arne Beekmann / Christoph Klimmt
}

Weblogs wird als besonders „authentischer" Form der Onlinekommunikation vielfach eine bohe Glaubwürdigkeit zugeschrieben. Immer häufiger versuchen Unternebmen, durch den Einsatz von sogenannten Corporate Blogs von dieser vermeintlich hoben Glaubwürdigkeit zu profitieren, denn glaubwürdige Kommunikation ist für erfolgreiche Öffentlichkeitsarbeit von essenzieller Bedeutung. Vor diesem Hintergrund wurde untersucht, ob in der Blogger-Community Unterschiede hinsichtlich der Glaubwürdigkeitsbeurteilung von Corporate Blogs und privaten Blogs bestehen. Dazu wurden in Anlebnung an Nawratil (1999) sechs Dimensionen von Glaubwürdigkeit unterschieden und mit einer quantitativen Online-Befragung von Blog-Nutzern in Bezug auf private und korporative Weblogs gemessen. Die Ergebnisse legen die Annabme nabe, dass Corporate Blogs bei den „Bloggern“ ein Glaubwürdigkeitsdefizit gegenüber privaten Blogs aufweisen. Befragte, die selbst private Blogs verfassen, standen der Glaubwürdigkeit sowohl korporativer wie privater Blogs nochmals etwas kritischer gegenüber als Personen, die (private und kommerzielle) Blogs nur lesen. Implikationen für die Online-PR und die weitere Forschung zu Weblogs werden diskutiert.

Schlüsselwörter: Weblog, Öffentlichkeitsarbeit, PR, Corporate Blog, Glaubwürdigkeit

\section{Problem}

Spätestens seitdem im September 2004 auf einem privaten Weblog in den USA ein Video auftauchte, in dem mit einem Kugelschreiber innerhalb weniger Sekunden ein Fahrradschloss des Herstellers Kryptonite geöffnet wurde, widmen viele PR-Experten der neuen Öffentlichkeit der Blogosphäre umfassende Aufmerksamkeit. Der Firma Kryptonite hat dieses Video, das sich zunächst in kürzester Zeit über verschiedenste Weblogs und später auch massenmedial verbreitete, einen Verlust von rund 10 Millionen US-Dollar beschert (Horowitz 2005). Große Aufmerksamkeit erreicht auch das deutsche Weblog Spreeblick mit seinem Beitrag über den Klingeltonhersteller Jamba (Scholz 2005: 30, hierzu auch Fischer 2006: 247ff.).

Diese Beispiele verdeutlichen, dass Weblogs mittlerweile durchaus ernst zu nehmende Informationsangebote sind, die von Rezipienten und Medien offensichtlich als brauchbare - mithin glaubwürdige - Quelle betrachtet werden und somit zur Meinungsbildung beitragen (vgl. Thimm/Berlinecke 2007, Zerfaß 2005b). Copeland (2004) ermittelte, dass die bessere Perspektive auf Themen, die Ehrlichkeit und die transparente Einseitigkeit zu den wichtigsten Gründen gehören, warum Weblogs von Internetusern besucht werden. Auch Blog-Autoren sehen Richtigkeit sowie Zuordbarkeit der Inhalte, Rechenschaftspflicht und Schadlosigkeit als Kriterien für ihre Arbeit (Cenite et al. 2009).

Mittlerweile investieren Unternehmen immer häufiger in eigene Corporate Blogs, um ihre kommunikativen Zielsetzungen auch im Internet zu realisieren (Eck 2007). Die vermeintlich hohe Glaubwürdigkeit, die Weblogs generell von Rezipienten und häufig auch von Experten zugeschrieben wird, lässt diese als besonders wirkungsvolles Instrument der Unternehmenskommunikation erscheinen (Röttger/Zielmann 2006: 41, Picot/ Fischer 2006: 3, Scholz 2005: 34). Während PR-Fachleute einen durch offene Kommunikationskultur geprägten Corporate Blog für ein geeignetes Mittel zur Stärkung von 
Authentizität halten (Lange 2006), stellt sich allerdings die Frage, ob den Corporate Blogs von den Rezipienten eine ähnlich hohe Glaubwürdigkeit zugesprochen wird wie den Weblogs, die keinen kommerziellen Absender haben. Denn Zerfaß/Bogosyan (2007) diagnostizieren einen deutlichen Glaubwürdigkeitsabfall gegenüber privaten Blogs.

Der vorliegende Beitrag stellt deshalb eine Studie vor, die sich mit der Glaubwürdigkeitszuschreibung von Corporate Blogs im Vergleich zu nicht kommerziellen Weblogs befasst. Im Gegensatz zu einer Vielzahl von Auftragsstudien zu Angebot, Nutzung und Bekanntheit von Weblogs sind kommunikationswissenschaftliche und insbesondere empirische Studien rar (vgl. Neuberger et al. 2007). Die Forschung zur Glaubwürdigkeit von Weblogs ist sowohl in Deutschland als auch international schwach ausgeprägt (ebd.). Einige wenige Studien (vgl. z. B. Johnson/Kaye 2004, Sonnabend 2005) attestieren Weblogs eine hohe Glaubwürdigkeit im Vergleich zu professionellem Journalismus. Ob diesen Anspruch auch Corporate Blogs für sich erheben können, ist bislang jedoch nicht angemessen untersucht worden.

Neben der kommunikationswissenschaftlichen Relevanz als Beitrag zur Grundlagenforschung über Onlinemedien im Allgemeinen sowie Weblogs und deren Glaubwürdigkeit im Speziellen zielt die Studie auch auf die Anwendungsimplikationen: Der erfolgreiche Austausch mit Blognutzern via Corporate Blogs ist abhängig von der wahrgenommenen Glaubwürdigkeit der Unternehmensbotschaften, da diese für Rezipienten ein wesentliches Selektionskriterium für die Zuwendung zu einem Medienangebot darstellt (Schweiger 1998: 123). Diese „Aufmerksamkeit der Bezugsgruppen im Sinne ihrer Hinwendung zu bestimmten Themen, Botschaften, Medien, Informationsangeboten und Kommunikatoren [ist für Unternehmen] eine unverzichtbare Voraussetzung jeder erfolgreichen Einflussnahme und Verständigung“ (Zerfaß 2004: 395).

Zudem sind Weblogs durch die Querverweise auf andere Blogs Teil eines dynamischen Kommunikationsraums. Etwaige Glaubwürdigkeitsdefizite von Unternehmensblogs könnten sich daher nicht nur negativ im Sinne der direkten Beurteilung durch ihre Leser auswirken, sondern gerade durch unabsehbare Multiplikations- und Verbreitungsprozesse in der Blogger-Community ernste Schäden für die Reputation von Unternehmen verursachen. Empirische Erkenntnisse zur Einschätzung der Glaubwürdigkeit von Unternehmensblogs sind demnach für den strategischen Einsatz dieses PRInstruments ausgesprochen bedeutsam.

\section{Gegenstand und Forschungsstand}

\subsection{Die Blogosphäre}

Die Möglichkeiten der Onlinekommunikation haben früh das Interesse von Akteuren im Bereich der Öffentlichkeitsarbeit geweckt (White/Raman 1999). Herkömmliche Websites gehören zum Standard-Repertoire moderner PR, und technische Innovationen werden zumeist zügig aufgegriffen. Dies gilt auch für Weblogs, welche Zerfaß (2005b: 4) folgend verstanden werden sollen als „persönliche oder thematische Nachrichtendienste, die mit Hilfe einfacher Content Management Systeme als Website im Internet publiziert, in regelmäßigen Abständen ähnlich wie ein Tagebuch um neue Einträge ergänzt und in vielfältiger Weise mit anderen Blogs und Websites verlinkt sind. Die Einträge sind umgekehrt chronologisch angeordnet und können jederzeit von den Nutzern kommentiert sowie mit Hilfe normierter Technologien in Echtzeit in andere Informationsangebote eingebunden bzw. zu neuen Diensten gebündelt werden.“

Die Anzahl der existierenden Weblogs ist schwer zu bestimmen, da nicht alle Angebote an eine der einschlägigen Plattformen angebunden sind. Spezielle Suchmaschinen 
wie etwa Technorati stoßen auf die Schwierigkeit, aktive von seit längerer Zeit nicht aktualisierten Blogs und Spam Blogs zu unterscheiden (Schmidt et al. 2009). Als Anhaltspunkt kann dienen, dass Technorati Anfang 2008 etwa 113 Millionen Blogs weltweit zählte (Cenite et al. 2009: 576). Auch die Größe der (von privaten Kommunikatoren dominierten) Blogosphäre ist schwer $\mathrm{zu}$ vermessen, zumal die Definitionen von Blognutzern variieren. Deisenberg berichtete 2007 auf der Basis repräsentativer Umfragedaten, dass eine Millionen Deutsche ein eigenes Weblog führen und ca. 2,5 Millionen Personen Weblogs besuchen. Die aktuelle ARD/ZDF-Online-Studie gibt an, dass acht Prozent der deutschen Online-Nutzer ab 14 Jahren (wobei rund 67 Prozent der Bevölkerung als Online-Nutzer gelten) Blogs mindestens „selten“ nutzen; etwa die Hälfte der Blognutzer wiederum bezeichnen sich als Leser und Verfasser, die andere Hälfte als reine Rezipienten. Damit ist die Anzahl der aktiven Blogger im Vergleich zu früheren Jahren deutlich gewachsen (Busemann/Gscheidle 2009). Parallel dazu findet eine Evolution der Blog-Technologien und Modi statt, beispielsweise der neuerdings populäre (und ebenfalls bereits von verschiedenen Unternehmen genutzte) Microblogging-Dienst „twitter“. Vor diesem Hintergrund ist anzunehmen, dass das Blog-Publikum eine relevante Größe aufweist und eine substanzielle Anzahl von nicht-kommerziellen Blogs selbst betreibt. Das Diffusionspotenzial gebloggter Informationen ist insofern erheblich (vgl. auch Schmidt 2006).

\subsection{Corporate Weblogs}

Corporate Blogs zeichnen sich im Gegensatz zu privaten Weblogs nach Zerfaß (2005b: 3) durch eine Bearbeitung durch Unternehmen bzw. deren Mitarbeiter aus (vgl. Kelleher/Miller 2006: 399). Prominente Beispiele sind u. a. der Blog von Frosta, der in der einschlägig orientierten Fachpresse häufig Lob erhielt, sowie die Blogs von Google, Payback und Doc Morris. Die Erscheinungsformen von Corporate Blogs sind vielfältig und bieten zahlreiche Möglichkeiten für die moderne Unternehmenskommunikation (vgl. Zerfaß 2005a, Kelleher/Miller 2006, Schwarzer et al. 2007). Corporate Blogs werden zum Beispiel zur Markenkommunikation, zur Unterstützung spezieller Kampagnen, im Kundenservice und technischen Support oder als sogenannte CEO-Blogs eingesetzt (Wolff 2006: 15). Letztere verfolgen in erster Linie das Ziel, „die wichtigsten Unternehmensvertreter bei den relevanten Bezugsgruppen bekannt zu machen und als glaubwürdige Repräsentanten darzustellen “ (Zerfaß/Sandhu 2006: 52f.). Zerfaß (2005a: 4) unterscheidet die Anwendungsmöglichkeiten von Corporate Blogs auf zwei Dimensionen, nämlich zum einen nach den Teilbereichen interne Kommunikation, Marktkommunikation und Public Relations und zum anderen nach der Vorgehensweise bei der Durchsetzung der Kommunikationsziele, die informativen, persuasiven oder argumentativen Charakter haben kann.

Trotz der kommunikativen Risiken wie Kontrollverlust des Kommunikators oder negative Kommentare anderer Blogger betonen PR-Experten vor allem die Chancen, die sich für Unternehmen in dieser neuen Öffentlichkeit der Weblogs ergeben: Zum einen ließen sich durch die Beobachtung der Beiträge aufkeimende Themen schneller identifizieren und dadurch die Reaktionszeit bei eventuellen Bedrohungen erheblich verkürzen. Zum anderen ermögliche das Betreiben eigener Weblogs eine dialogorientierte, authentische, schnelle und ungefilterte Kommunikation mit wichtigen Bezugsgruppen (Zerfaß 2005b: 3f.). In der Glaubwürdigkeit wird der größte Vorteil gesehen (Röttger/ Zielmann 2006: 41); sie dürfte der Grund für den vermehrten Einsatz von Weblogs als Instrument der Unternehmenskommunikation sein (vgl. auch Picot/Fischer 2006: 3, Scholz 2005: 34, Yang/Lim 2009). 
Nach Eck (2007) existierten 2007 mehr als 600 verschiedene Unternehmensblogs in Deutschland. Systematisch erhobene Daten liegen aber nicht vor. Die von Eck dokumentierte Entwicklung ist jedoch beachtlich, zumal die Anzahl von Corporate Blogs im Sommer 2004 noch auf lediglich zwei Dutzend geschätzt wurde (Pleil 2004: 11).

\subsection{Glaubwürdigkeitsforschung}

Es ist das oberste Ziel der Unternehmenskommunikation, „die Meinungen, Einstellungen oder Verhaltensweisen der Stakeholder zu beeinflussen oder zu verändern“ (Mast 2006: 50). Dies spricht dafür, der Glaubwürdigkeit besondere Beachtung zu schenken, denn schon Hovland und Weiss (1951: 642f.) stellten fest, dass Quellen, denen eine hohe Glaubwürdigkeit attestiert wurde, signifikant häufiger Meinungsänderungen herbeiführten als Quellen, die weniger glaubwürdig beurteilt wurden. Insbesondere Organisationen, die häufig über Medien an die Gesellschaft herantreten, sind auf die Zuschreibung öffentlichen Vertrauens angewiesen, weil der Wahrheitsgehalt einer medial vermittelten Information in der Regel nicht direkt und unmittelbar nachprüfbar ist (Bentele/ Seidenglanz 2005: 346, Köhnken 1990: 1).

Glaubwürdigkeit als Ziel und Erfolgsgröße unternehmerischer Öffentlichkeitsarbeit ist unter anderem wegen des kommerziellen Hintergrunds von Unternehmen oftmals nur schwer zu erreichen (Hubig/Siemoneit 2007). Auch das Instrument der Corporate Weblogs muss in Hinsicht auf die ihm zugeschriebene Glaubwürdigkeit kritisch betrachtet werden. So betonen Yang und Lim (2009) die Bedeutung der Glaubwürdigkeit blogbasierter PR für die Vertrauensbildung beim Publikum und damit den PR-Erfolg insgesamt.

Zerfaß/Bogosyan (2007) ermittelten in ihrer Blogstudie, dass etwa ein Viertel der Befragten den Inhalten von Corporate Blogs nicht trauen. Allerdings wird diese Untersuchung der Multidimensionalität der rezipientenseitigen Glaubwürdigkeitszuschreibung nicht gerecht (vgl. u. a. Jacobson 1969, McCroskey/Jenson 1975, Gaziano/McGrath 1986, Wirth 1999). Kelleher und Miller (2006) schließen aus einem Experiment, dass ein Corporate Blog eher als herkömmliche Websites in der Lage sei, einer Unternehmung eine „menschliche Stimme“ zu verleihen und die wahrgenommene Anonymität einer Großorganisation zu reduzieren - damit könnten Zugewinne an wahrgenommener Glaubwürdigkeit verbunden sein, auch wenn dies nicht explizit untersucht wurde. Die bisherigen wissenschaftlichen Studien zur Glaubwürdigkeit von Corporate Blogs sind insgesamt insofern unzureichend, weil das Konstrukt Glaubwürdigkeit konzeptionell $\mathrm{zu}$ wenig elaboriert und in verengter Weise operationalisiert wurde. Daher rekurriert der vorliegende Beitrag auf die Forschung zu journalistischer Glaubwürdigkeit, um daraus forschungsleitende Überlegungen in Bezug auf Corporate Blogs zu gewinnen.

Bentele (1988: 408) ist der Auffassung, dass die Glaubwürdigkeitsbeurteilung einer Quelle das Ergebnis eines Zuschreibungsprozesses ist. Er bestimmt Glaubwürdigkeit „als eine Eigenschaft, die Menschen, Institutionen oder deren kommunikativen Produkten (mündliche oder schriftliche Texte, audiovisuelle Darstellungen) von jemandem (Rezipienten) in Bezug auf etwas (Ereignisse, Sachverhalte usw.) zugeschrieben wird.“ Daraus ergibt sich eine „prinzipielle Bereitschaft [...], Botschaften eines bestimmten Objektes als zutreffend $\mathrm{zu}$ akzeptieren und bis zu einem gewissen Grad in das eigene Meinungs- und Einstellungsspektrum zu übernehmen." (Wirth 1999: 55)

Hovland und Weiss (1951) verstanden Glaubwürdigkeit zunächst als zweidimensionales Konstrukt, das sich aus Kompetenz und Vertrauenswürdigkeit zusammensetzt. Methodisch konsequent entstanden aus diesem Ansatz insbesondere in den 60er und 70er Jahren zahlreiche Studien, die mithilfe von semantischen Differentialen die Mehr- 
dimensionalität der Glaubwürdigkeitszuschreibung erforschten (Wirth 1999: 48). Dieser zumeist faktorenanalytische Ansatz der Glaubwürdigkeitsmessung brachte zahlreiche, aber teilweise sehr unterschiedliche Dimensionierungen hervor (vgl. z. B. Jacobson 1969, Mosier/Ahlgreen 1981; für einen Überblick Nawratil 1997, Wirth 1999). Der disparate Forschungsstand ist sicherlich ein wichtiger Grund dafür, dass die Glaubwürdigkeit von Kommunikationsangeboten häufig entweder noch unidimensional gemessen oder auf eine scheinbar willkürliche Anzahl an Dimensionen - in der Regel Kompetenz und Vertrauenswürdigkeit - reduziert wird (Wirth 1999: 48). Des Weiteren scheinen viele der Adjektivpaare, die zur Messung mittels semantischer Differentiale eingesetzt werden, nur schwer auf Medienangebote anwendbar, da sie häufig personenbezogene Charaktereigenschaften ausdrücken (vgl. z. B. Jacobson 1969; für einen Überblick Wirth 1999).

Nawratil (1999) hat einen Ansatz erarbeitet, der die nach ihrer Argumentation relevanten sechs Glaubwürdigkeitsdimensionen speziell auf Medienangebote überträgt und spezifiziert. Sie unterscheidet die folgenden Dimensionen:

- Kompetenz: Diese Dimension „manifestiert sich [...] im Ausmaß des Wissens um ein bestimmtes Fachgebiet, in Hinweisen auf Qualifikation, Leistungen oder Intelligenz.“ (Nawratil 1999: 17) Die Fachkompetenz einer Person bezüglich des Wissens um eine bestimmte Sache, Kenntnisse, Fähigkeiten oder gar deren Äußerung in Berufsbezeichnungen und akademischen Titeln, interpretiert Nawratil (1999: 25) bei Medien als Vermittlungskompetenz, also als sachgerechten Umgang mit Quellen und deren Mitteilungen. Zu dieser „Einhaltung der journalistischen Handwerksregeln“ (ebd.: 27) zählt „die Beantwortung der sogenannten W-Fragen ebenso wie die Auswahl der relevanten Quellen und ihrer Aussagen, die korrekte Vorstellung der Aussageträger sowie eine angemessene Transformation der Aussage, die den Sinnkern nicht entstellt.“ (ebd.: 25)

- Vertranenswürdigkeit: Diese Glaubwürdigkeitsdimension gibt „Aufschluss darüber, ob und inwieweit ein Ausgangspartner oder Bewertungsobjekt hinsichtlich seiner Aussagen und Handlungen verlässlich ist" (Nawratil 1997: 226). Vertrauenswürdigkeit konstituiert sich bei Personenquellen vor allem durch die schon von Aristoteles benannten Subdimensionen Ehrlichkeit (Rechtschaffenheit, Gesetzestreue) und Lauterkeit (Reinheit der Motive). Nawratil (1999: 25) zufolge konkretisiert sich eine vertrauenswürdige Quelle auf Seiten der Medien durch Objektivität, Fairness und Unparteilichkeit. Unter Fairness können in diesem Zusammenhang die Forderungen nach unverfälschter Wiedergabe von Inhalten und der Trennung von Nachricht und Meinung gebündelt werden. Unparteilichkeit meint Unabhängigkeit im Sinne der Abwesenheit von Eigeninteressen. Objektivität zeigt sich durch die Ausgewogenheit der Positionen, falls mehrere Meinungen vertreten werden.

- Dynamik: Nach Berlo, Lemert und Mertz (1969: 575f.) ist Dynamik als wertende Dimension zu interpretieren, die der Quelle zusätzliche Intensität verleihen kann: „...given an evaluation of a source as safe or unsafe, qualified or unqualified, the polarity or intensity of these evaluations of the source is intensified through perceptions of high dynamism“. Die Stil- und Gestaltungselemente einer Botschaft oder eines Kommunikators (vgl. Wirth 1999: 50) lassen sich auch auf Medien übertragen und zeigen sich in Aufmachung und Präsentation (Nawratil 1999: 26) und Merkmalen wie Typographie und Layout einer Zeitung oder der formalen Gestaltung von Nachrichtensendungen des Rundfunks (Bentele 1988: 422).

- Soziale Billigung: Als weiterer Faktor kann die Mehrheitsmeinung über den Aussageträger oder das Bewertungsobjekt (vgl. Giffin 1967) angesehen werden. „Ob jemand Anerkennung erfährt oder als abgelehnter Außenseiter gilt, hat Einfluß auf 
seine Glaubwürdigkeit, wobei zusätzlich in Betracht zu ziehen ist, von welcher Seite einer Quelle Ablehnung entgegenschlägt oder Zustimmung zuteilwird." (Nawratil 1999: 19) Die wahrgenommene Meinung Dritter, die sich in Akzeptanz oder Ablehnung einer Person äußert, ist auch bei Medien zu beobachten. So wäre denkbar, dass das Glaubwürdigkeitsdefizit von (Boulevard-)Kaufzeitungen auch auf die fehlende soziale Billigung zurückzuführen ist, die bei Prestigemedien hingegen stark ausgeprägt ist (Nawratil 1999: 26; vgl. auch Meyer 1988).

- Ähnlichkeit: Die (wahrgenommene) Ähnlichkeit zwischen dem Kommunikator und dem Publikum spielt eine wichtige Rolle für die Glaubwürdigkeitsbeurteilung (vgl. Simons et al. 1970). Diese kann sich sowohl auf die Gruppenzugehörigkeit als auch auf Einstellungen und Wertehaltungen beziehen (Lowry 1973: 195). „Trotz des Einflusses der Ähnlichkeit auf Meinungs- und Einstellungsänderungen kann häufig nur ein geringer Einfluß auf die wahrgenommene Glaubwürdigkeit nachgewiesen werden“ (Nawratil 1999: 18). Somit kommt der Ähnlichkeit von Kommunikator und Publikum eher „der Rang eines Zusatzkriteriums zu, das sich nur dann auf die Glaubwürdigkeit auswirkt, wenn andere Kriterien, insbesondere Hinweise auf die Kompetenz, fehlen“ (ebd.; vgl. dazu auch Simons et al. 1970). Medienspezifisch spiegelt sich Ähnlichkeit in der Nähe der publizistischen Linie zu den Wertehaltungen der Rezipienten (Nawratil 1999: 26). Bezogen auf Weblogs, die im Gegensatz zu vielen herkömmlichen Internetseiten meistens von Einzelpersonen oder kleinen Gruppen verantwortet werden (Neuberger 2005: 79), sind Ähnlichkeiten allerdings eher in den personenbezogenen Unterdimensionen Gruppenzugehörigkeit sowie Einstellungen und Wertehaltung zu vermuten (vgl. Nawratil 1999: 18).

- Sympathie: Sympathie kann als Zusatzkriterium der Glaubwürdigkeitsbeurteilung betrachtet werden (vgl. McCroskey/Jenson 1975), auch wenn dieser Zusammenhang nicht immer eindeutig nachzuweisen ist (Nawratil 1997: 235). In der interpersonalen Kommunikation konstituiert sich Sympathie aus der wahrgenommenen Attraktivität des Kommunikators (Nawratil 1999: 18), aber auch aus einer emotionalen Nähe, Umgänglichkeit und Humor (ebd.: 27). Eine emotionale Nähe kann auch zu Medien bestehen (ebd.). Anwendbare Subdimensionen sind positive Wertschätzung (Forgas 1987: 229), angenehmes Empfinden (Pelz/Scholl 1990: 34), ähnliche Interessen (vgl. Wheeless 1976: 75f.) und Intelligenz (vgl. Byrne 1961).

Diese Konstruktdimensionalisierung von Nawratil (1997) wurzelt in der Journalismusforschung und nicht in der Forschung zur Unternehmenskommunikation. Entsprechend stellt sich die Frage, ob die Dimensionseinteilung zielführend für die vorliegende Fragestellung ist. Hier wäre einerseits zu argumentieren, dass journalistische und PRBotschaften potenziell nicht den gleichen (Glaubwürdigkeits-)Anforderungen unterliegen und ergo ein PR-spezifischeres Verständnis von Glaubwürdigkeit notwendig wäre. Dem steht das Argument gegenüber, dass Weblogs in inhaltlicher Hinsicht Parallelen zu jüngeren Stilformen des Journalismus' aufweisen und auch als mögliche Konkurrenz oder Ergänzung zu konventionellem Journalismus diskutiert werden (z. B. Neuberger et al. 2007). Vor diesem Hintergrund wäre eine Anwendung journalismusorientierter Glaubwürdigkeitsdimensionen auf den Gegenstand von Corporate Weblogs durchaus angemessen. Schließlich ist zu bedenken, dass die Literatur zur Unternehmenskommunikation zwar einhellig auf die Bedeutung von Glaubwürdigkeit verweist (z. B. Hubig/ Siemoneit 2007), dass aber PR-spezifische und konzeptionell tragfähige Dekonstruktionen des komplexen Glaubwürdigkeitskonstrukts in der thematischen Literatur kaum zu finden sind. Insgesamt erscheint daher die Dimensionalisierung von Nawratil als 
gangbarer Weg, um die Glaubwürdigkeit von Corporate Weblogs empirisch zu untersuchen.

\subsection{Die Glaubwürdigkeit von Corporate Blogs: Integration und Hypothesen}

Die bisherigen Überlegungen verdeutlichen, dass der Glaubwürdigkeitszuspruch der Rezipienten für Corporate Blogs von essenzieller Bedeutung ist, um diese als Instrumente der Unternehmenskommunikation erfolgreich einzusetzen. Die informelle, subjektiv-persönliche und spontan wirkende Charakteristik von Weblogs (z. B. Thimm/ Berlinecke 2007, Yang/Lim 2009) könnte Corporate Blogs in den Augen der Leser glaubwürdiger erscheinen lassen als andere Werkzeuge der (Online)-PR. Wegen der Multidimensionalität von Glaubwürdigkeit lassen sich hinsichtlich einzelner Aspekte aber auch Defizite von Corporate Blogs vermuten: So ist ein kommerzielles Eigeninteresse bei korporativer Kommunikation inhärent (Mast 2006: 50), was sich auf die Beurteilung der Vertrauenswürdigkeit negativ auswirken könnte (Callison 2001). Andererseits gilt das Medium Weblog ohnehin als „transparent einseitig“ (Copeland 2004), was dieses Vertrauensdefizit relativieren könnte. Ein kommerzieller und persuasiver Charakter könnte zudem die Dimensionen Sympathie und wahrgenommene Ähnlichkeit sowie die allgemeine soziale Billigung negativ beeinflussen. Lediglich hinsichtlich der Dimensionen Kompetenz und Dynamik ist aufgrund der Professionalität und umfangreichen Ressourcenbasis von Corporate Blogs kein Defizit - oder womöglich sogar eine Überlegenheit - gegenüber nicht-kommerziellen Blogs zu erwarten. Entsprechend wird dimensionsspezifisch eine Hypothese aufgestellt und eine Forschungsfrage formuliert:

H1: Blogs privater Autoren/innen werden von Blognutzern/innen glaubwürdiger als Corporate Blogs eingeschätzt im Sinne von vertrauenswürdiger, sympathischer, ähnlicher und sozial eher gebilligt.

F1: Wie werden Corporate Blogs von Internetnutzer/inne/n hinsichtlich ibrer Glaubwürdigkeit im Sinne von Kompetenz und Dynamik im Vergleich zu privaten Blogs beurteilt?

Neben einer Differenzierung nach Glaubwürdigkeitsdimensionen, die einzeln in Bezug auf Corporate Blogs zu untersuchen sind, bietet sich auch eine Spezifizierung der Perspektive auf die Zielgruppen von Corporate Blogs an. Denn die kommunikative Bedeutung von Weblogs ergibt sich speziell durch die Querverbindungen unterschiedlicher Blogs, die eine netzwerkinduzierte schnelle und weitreichende Diffusion von Nachrichten und Meinungen ermöglichen (vgl. Schmidt 2006; Thimm/Berlinecke 2007). Insofern ist es relevant zu wissen, inwiefern Personen, die selbst aktiv bloggen und damit potenziell zur Weiterverbreitung von Informationen aus Corporate Blogs beitragen, die Glaubwürdigkeit von Corporate Blogs einschätzen. Unter dem Gesichtspunkt des Diffusionspotenzials von Bloginformation repräsentieren die aktiven Blogger die Netzwerkknoten, denen Online-PR besondere Aufmerksamkeit schenken muss (vgl. Zerfaß 2005b). Wenn diese Gruppe Corporate Blogs aufgeschlossen gegenüber steht (oder gerade besonders kritisch über deren Glaubwürdigkeit urteilt), ergeben sich konkrete Implikationen für den strategischen Wert und die Ausgestaltung von Corporate Blogs. Als Vergleichsbasis bieten sich Leser von Corporate Blogs an, die keinen eigenen Blog unterhalten und damit zwar Teil des Publikums von Online-PR sowie potenzielle Multiplikatoren außerhalb der Blogosphäre sind, aber eben nicht als Verbreiter im Blogverbund tätig werden. Hierzu wird keine Annahme aufgestellt, sondern vielmehr eine weitere Forschungsfrage abgeleitet:

F2: Wie unterscheiden sich aktive Blogger von reinen Bloglesern hinsichtlich ibrer Glaubwürdigkeitsurteile über Corporate Blogs? 
Schließlich ist zu überlegen, inwiefern die Glaubwürdigkeit von Corporate Blogs durch eine generelle Skepsis gegenüber Wirtschaftsunternehmen im Allgemeinen bedingt ist. Sollten Corporate Blogs unabhängig von allgemeiner Unternehmens-Skepsis (im Sinne kritischer Einstellungen gegenüber kapitalistischen Unternehmen, vgl. NoelleNeumann/Köcher 1997: 677) als weniger glaubwürdig eingestuft werden, würde dies Corporate Blogs als Kommunikationskanal für die Online-PR viel deutlicher in Frage stellen als eine reduzierte Glaubwürdigkeitszuschreibung, die auf allgemeine Skepsis gegenüber Unternehmen und ihrer Kommunikation (vgl. Callison 2001) zurückzuführen ist. Entsprechend wird eine weitere Forschungsfrage formuliert:

F3: Wie hängt die Einstellung zu Unternehmen mit der Glaubwürdigkeitszuschreibung zu Corporate Blogs zusammen?

\section{Methode}

\section{1 Überblick}

Um die genannte Hypothese zu testen und die Forschungsfragen zu beantworten, wurde vom 23.07.2007 bis zum 05.08.2007 eine quantitative Online-Befragung durchgeführt. Sie richtete sich an Rezipienten wie Autoren von Blogs. Die Teilnehmer waren gebeten, jeweils einen Frageblock zu Corporate Blogs und einen zu privaten Blogs auszufüllen. Um dem Probanden den Unterschied zwischen Corporate Blogs und privaten Blogs zu verdeutlichen, war auf jeder Seite des jeweiligen Fragenblocks eine entsprechende einfache Definition - angelehnt an die oben zitierte Begriffsbestimmung - sichtbar. Um Reihenfolgeeffekte zu vermeiden, wurden sowohl die zwei Fragenblöcke als auch die Aussagen in den Itembatterien per Zufall über die Probanden hinweg rotiert.

\subsection{Inbalte des Fragebogens}

In den bisherigen Studien zur Glaubwürdigkeit von Medienangeboten wurden häufig semantische Differenziale mit Adjektivpaaren eingesetzt, die in vorherigen Untersuchungen durch Faktorenanalysen als Indikatoren identifiziert wurden (vgl. Jacobson 1969, Singletary 1976, Mosier/Ahlgren 1981, Austin/Dong 1994; für einen Überblick Wirth 1999). Matthes/Kohring (2003: 7f.) kritisieren diese abstrakte Vorgehensweise, weil die Adjektivpaare lediglich allgemeine Charakteristika von Kommunikation beschreiben. Sie plädieren deshalb für eine Übersetzung in konkrete forschungsspezifisch formulierte Items. Zur Konkretisierung der Glaubwürdigkeitsdimensionen nach Nawratil (1997; vgl. oben 2.3) auf Weblogs wurden diese deshalb in Subskalen mit jeweils vier Items operationalisiert. Diese wurden den Befragten einmal zur Einschätzung in Bezug auf private Blogs ohne kommerziellen Hintergrund und ein weiteres Mal zur Beantwortung mit Blick auf Corporate Blogs vorgelegt. Dabei sollten die Bewertungen auf Grundlage der jeweils subjektiven Erfahrungen der Probanden mit diesen beiden Arten von Weblogs getroffen werden. Der Einsatz von Stimulus-Weblogs hätte Ergebnisse generiert, die sich nicht auf die allgemeine Einstellung, sondern auf konkrete Beispiele beziehen. Das Problem der Unklarheit, welche Arten von Blogs die Probanden jeweils vor Augen hatten, wurde zugunsten einer Generalisierbarkeit auf verschiedene Typen und Inhalte von Blogs in Kauf genommen. So können allerdings keine Aussagen über spezifische Angebotsarten getroffen werden. 
Tabelle 1: Interne Konsistenzen der Items zu den Glaubwürdigkeitsdimensionen

\begin{tabular}{lcc}
\hline Cronbach's Alpha & Corporate Blogs & Private Blogs \\
\hline Kompetenz (4 Items) & .87 & .85 \\
Vertrauenswürdigkeit (4 Items)* & .48 & .23 \\
Dynamik (4 Items) & .78 & .76 \\
Ähnlichkeit (3 Items) & .76 & .69 \\
Soziale Billigung (4 Items) & .75 & .77 \\
Sympathie (4 Items) & .84 & .86 \\
\hline
\end{tabular}

$\mathrm{N}=205$

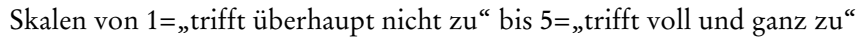

*zwei Variablen wurden zur Reliabilitätsprüfung invertiert

Mit Ausnahme der Dimension Vertrauenswürdigkeit wiesen die Subskalen für die einzelnen Dimensionen der Glaubwürdigkeit akzeptable interne Konsistenzen auf und konnten daher zu Indices verdichtet werden (Tab. 1). Die Items zur Dimension Vertrauenswürdigkeit ergaben hingegen keinen homogenen Index ${ }^{1}$, so dass hier auf das Einzelitem „Es ist in der Regel deutlich zu erkennen, ob es sich um Fakten oder um die Meinung des Bloggers handelt“ zurückgegriffen wurde, um diese Dimension empirisch abzubilden. Die Operationalisierung der Glaubwürdigkeitsdimensionen kann deshalb nur mit Einschränkung als gelungen gelten.

Zur Beantwortung von F3 wurde ferner die Einstellung zu Unternehmen mit Hilfe von acht Items mit 5er-Skalierung (nach Noelle-Neumann/Köcher 1997, z. B. „Die meisten Unternehmen beuten ihre Mitarbeiter aus“) abgefragt, wobei der Wert „1“ „stimme überhaupt nicht $\mathrm{zu}$ “ und der Wert „5“ „stimme voll und ganz zu“ bedeutete. Die Itembatterie erwies sich als hinreichend homogen (Cronbach's $\alpha=.84$ ) und wurde $\mathrm{zu}$ einem Mittelwertindex verdichtet. Aktive Blogger, also Personen, die ein eigenes Weblog schreiben, standen Unternehmen signifikant kritischer gegenüber $(M=2,79$, $\mathrm{SD}=0,77)$ als Personen, die lediglich Weblogs lesen $(\mathrm{M}=3,25, \mathrm{SD}=0,70, \mathrm{t}(205)=-4,381$, $\mathrm{p}<.01)$.

Es ist anzumerken, dass zwar nicht alle Corporate Blogs, wohl aber der überwiegende Teil von Wirtschaftsunternehmen stammt. In Bezug auf den zu untersuchenden $\mathrm{Zu}-$ sammenhang zwischen Vorbehalten gegenüber Wirtschaftsunternehmen und der Einstellung zu Corporate Blogs wurde nicht zwischen verschiedenen Blog-Kommunikatoren unterschieden. Diese Unschärfe und die darauf folgende möglicherweise geringere Varianzaufklärung wird jedoch in Kauf genommen, weil die Studie generalisierbare Aussagen über alle Formen von Corporate Blogs treffen will und eine differenzierte Analyse für Subtypen nachfolgenden Studien überlässt (vgl. auch unten 5.).

Der Fragebogen enthielt neben der Operationalisierung der relevanten Konstrukte auch allgemeine Fragen zur Nutzung von Corporate und privaten Blogs als Leser und als Autor sowie zu demografischen Aspekten.

\subsection{Rekrutierungsstrategie und Stichprobe}

Da die Glaubwürdigkeit von kommerziellen und nicht-kommerziellen Weblogs nur von Nutzern dieser Online-Angebote beurteilt werden kann, wurden nur solche Probanden in

1 Die mangelnde Reliabilität kann vermutlich darauf zurückgeführt werden, dass diese Subskala als einzige zwei negativ formulierte Items enthielt. 
die Stichprobe aufgenommen, die zum Zeitpunkt der Befragung mindestens ein kommerzielles und ein nicht-kommerzielles Weblog genutzt hatten. Um die auf die Gesamtbevölkerung gesehen noch relativ kleine Gruppe von Bloggern mit vertretbarem Aufwand gezielt ansprechenzukönnen, wurdefür dieTeilnahme ander Befragung aufverschiedenen Wegen geworben: Autoren unterschiedlichster Blogs wurden kontaktiert und gebeten, auf die Studie aufmerksam zu machen ${ }^{2}$. Weitere Befragte wurden im E-Mail-Schneeballverfahren gewonnen. E-Mails wurden mit der Bitte um Weiterleitung an universitäre Medienund Informatikinstitute verschickt, weil unter deren Studierenden eine hohe BloggerAnzahl vermutet wurde.

Es handelt sich um eine selbstselektive Online-Stichprobe, die daher keine Repräsentativität für die deutschsprachige Blogger-Community beanspruchen kann. Für den Glaubwürdigkeitsvergleich von privaten und Corporate Blogs kann die Studie dennoch vorläufige Aussagekraft beanspruchen.

Die Fragebogen-Website wurde insgesamt 1370-mal aufgerufen, die Hälfte brach die Befragung bereits auf der ersten Seite ab, was auf den dortigen Hinweis zurückzuführen sein dürfte, dass man für die Teilnahme sowohl ein privates als auch ein UnternehmensBlog genutzt haben musste.

Personen, die nach eigener Angabe selbst Corporate Blogs schreiben, wurden bei der Auswertung ausgeschlossen. Denn solche Personen müssen als Spezialisten für OnlinePR gelten, die sich hinsichtlich ihrer Vorbildung, Kommunikationsziele (beim Bloggen und bei der Beantwortung des Fragebogens) und allgemeinen Perspektive auf den Gegenstand systematisch von der „allgemeinen Blogosphäre“ unterscheiden. In diesem Sinne würden aktive Corporate Blogger zu einer komplizierteren Datenlage beitragen.

Insgesamt gingen die Daten von 205 Befragten in die Analyse ein. Die Stichprobe bestand zu 75 Prozent aus Männern, das durchschnittliche Alter lag bei 31 Jahren. Die Teilnehmer waren zwischen 16 und 77 Jahren alt, wobei etwa drei Viertel der Befragten 36 Jahre oder jünger waren. Rund 85 Prozent der Teilnehmer verfügten mindestens über Fachhochschulreife. Zwischen Personen, die selbst Weblogs schreiben ( $n=79 ; 39 \%)$ und Personen, die lediglich Weblogs lesen ( $\mathrm{n}=126 ; 62 \%)$, ließen sich hinsichtlich der soziodemografischen Merkmale kaum Unterschiede feststellen.

Die von den Teilnehmern geschätzte wöchentliche Nutzungsdauer privater Weblogs lag bei durchschnittlich 210 Minuten ( $\mathrm{SD}=315,2)$; immerhin rund 16 Prozent nutzten private Blogs wöchentlich sieben Stunden oder länger. Corporate Blogs widmeten sich die Befragten durchschnittlich nur 35 Minuten $(\mathrm{SD}=90,8)$. Allerdings finden sich auch Intensivnutzer, acht Prozent verbrachten wöchentlich zwei Stunden oder länger mit Corporate Blogs. Die Anzahl genutzter Weblogs in den letzten drei Monaten vor der Befragung lag bei durchschnittlich 31 privaten Blogs $(\mathrm{SD}=58,4)$. Dagegen wurden in den letzten drei Monaten vor der Befragung nur durchschnittlich acht Corporate Blogs $(\mathrm{SD}=22,3)$ von den Befragten gelesen.

\section{Ergebnisse}

Zur Prüfung der Hypothese und Beantwortung der ersten beiden Forschungsfragen wurde je Glaubwürdigkeitsdimension eine Varianzanalyse mit Messwiederholung durchgeführt. Dabei ging jeweils die Beurteilung der Glaubwürdigkeit von Corporate Blogs und von privaten Blogs als Innersubjektfaktor ein (da alle Befragten Glaubwür-

2 Auf die Umfrage wurde beispielsweise auf den Blogs http://www.moderne-unternehmenskommunikation.de, http://www.mattwagner.de/2007/07/fundstcke-34.htm, http://www.werbeblog ger.de und http://dittes.info/verlinkt. 
digkeitsurteile für beide Blogtypen abgegeben hatten). Die Klassifikation als reiner Blogleser oder aber Blogautor und -leser wurde zudem als Zwischensubjektfaktor eingegeben (Tab. 2).

Tabelle 2: Deskriptiv- und Inferenzstatistik zum Vergleich von Corporate Blogs und privaten Blogs hinsichtlich der mehrdimensionalen Glaubwürdigkeitszuschreibung von Blogverfassern und reinen Bloglesern

\begin{tabular}{|c|c|c|c|c|c|c|c|}
\hline \multirow{2}{*}{$\begin{array}{l}\text { Dimensi- } \\
\text { on }\end{array}$} & \multicolumn{3}{|c|}{ Corporate Blogs } & \multicolumn{3}{|c|}{ Private $\mathrm{B} \log s$} & \multirow{2}{*}{ 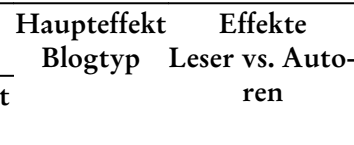 } \\
\hline & $\begin{array}{c}\text { Blogle- } \\
\text { ser }\end{array}$ & $\begin{array}{l}\text { Blogauto- } \\
\text { ren }\end{array}$ & Gesamt & $\begin{array}{l}\text { Blogle- } \\
\text { ser }\end{array}$ & $\begin{array}{l}\text { Blogauto- } \\
\text { ren }\end{array}$ & Gesamt & \\
\hline $\begin{array}{l}\text { Kompe- } \\
\text { tenz }\end{array}$ & 2,54 & 2,41 & 2,49 & 3,24 & 2,97 & 3,14 & $\begin{array}{c}\mathrm{F}(1,203)=57, \text { Haupteffekt: } \\
\mathrm{p}<.01, \text { par- } \mathrm{F}(1,203)=4,09, \\
\text { tielles } \eta^{2}=.22 \mathrm{p}<.05, \text { partiel- } \\
\text { les } \eta^{2}=.02\end{array}$ \\
\hline $\begin{array}{l}\text { Vertrau- } \\
\text { ens-wür- } \\
\text { digkeit } \\
\text { (Einzel- } \\
\text { Item) }\end{array}$ & 2,90 & 2,77 & 2,84 & 3,59 & 3,61 & 3,60 & $\begin{array}{l}\mathrm{F}(1,203)=51, \quad \text { Nein } \\
\mathrm{p}<.01, \text { par- } \\
\text { tielles } \eta^{2}=.20\end{array}$ \\
\hline Dynamik & 3,29 & 3,16 & 3,24 & 3,33 & 3,25 & 3,30 & $\mathrm{~F}<1, \mathrm{~ns}$ \\
\hline $\begin{array}{l}\text { Ähnlich- } \\
\text { keit }\end{array}$ & 2,59 & 2,43 & 2,53 & 3,44 & 3,30 & 3,39 & $\begin{array}{cc}\mathrm{F}(1,203) & \text { Haupteffekt: } \\
=148, & \mathrm{~F}(1,203)=2,78, \\
\mathrm{p}<.01, \text { par- } & \mathrm{p}<.10 \text {, partiel- } \\
\text { tielles } \eta^{2}=.42 & \text { les } \eta^{2}=.01\end{array}$ \\
\hline $\begin{array}{l}\text { Soziale } \\
\text { Billigung }\end{array}$ & 2,61 & 2,62 & 2,61 & 3,57 & 3,48 & 3,53 & $\begin{array}{l}\mathrm{F}(1,203) \quad \text { Nein } \\
\quad=121, \\
\mathrm{p}<.01, \text { par- } \\
\text { tielles } \eta^{2}=.37\end{array}$ \\
\hline $\begin{array}{l}\text { Sympa- } \\
\text { thie }\end{array}$ & 2,73 & 2,63 & 2,69 & 3,82 & 3,90 & 3,85 & $\begin{array}{c}\mathrm{F}(1,203) \\
=173, \\
\mathrm{p}<.01, \text { par- } \\
\text { tielles } \eta^{2}=.46\end{array}$ \\
\hline
\end{tabular}

$\mathrm{N}=205$

Skalen von 1 = „(jeweilige Subdimension der Glaubwürdigkeit) trifft überhaupt nicht zu“ bis $5=$ „trifft voll und ganz zu“

Die Befunde zeigen, dass Corporate Blogs auf mehreren Dimensionen deutlich weniger glaubwürdig eingeschätzt werden als private Blogs; bei den Dimensionen Vertrauenswürdigkeit und Kompetenz fällt der Unterschied etwas kleiner aus als bei der sozialen Billigung, Ähnlichkeit und Sympathie. Hinsichtlich der Dimension Dynamik ergeben sich keine Unterschiede zwischen privaten und Corporate Blogs. Die Befunde bestätigen damit H1 weitgehend und weisen auf Glaubwürdigkeitsunterschiede bei der Kompetenz-, nicht aber der Dynamik-Dimension hin (F1). Über alle Dimensionen hinweg (mit Ausnahme der sozialen Billigung) beurteilen die Personen, die selbst Blogs verfassen, die Glaubwürdigkeit von Corporate Blogs kritischer als Personen, die Blogs nur lesen, wobei diese Unterschiede nur schwach ausgeprägt sind.

Zur Beantwortung der dritten Forschungsfrage wurde eine Korrelationsmatrix erstellt, die die Zusammenhänge zwischen der Einstellung zu Unternehmen (Mittelwert- 
index, höhere Werte repräsentieren eine positivere Einstellung zu Unternehmen) und der empfundenen Glaubwürdigkeit von Corporate Blogs nach Dimensionen zeigt (Tab. 3).

\section{Tabelle 3: Zusammenhang zwischen Einstellung zu Unternebmen und zugeschriebener Glaubwürdigkeit von Corporate Blogs}

\begin{tabular}{lcc}
\hline $\begin{array}{l}\text { Dimension (Einschätzung in Bezug auf } \\
\text { Corporate Blogs)* }\end{array}$ & $\begin{array}{c}\text { Korrelation mit Einstellung zu } \\
\text { Unternehmen** (Pearson’s r) }\end{array}$ & Signifikanz \\
\hline Kompetenz (4 Items) & .214 & .002 \\
Vertrauenswürdigkeit (Einzelitem) & .150 & .038 \\
Dynamik (4 Items) & .173 & .013 \\
Ähnlichkeit (3 Items) & .185 & .008 \\
Soziale Billigung (4 Items) & .095 & $\mathrm{~ns}$ \\
Sympathie (4 Items) & .146 & .037 \\
\hline
\end{tabular}

$\mathrm{N}=205$

*Skalen von 1 = „(jeweilige Subdimension der Glaubwürdigkeit) trifft überhaupt nicht zu“ bis 5 = „trifft voll und ganz zu“

* Skala von $1=$ „(Unternehmen-befürwortende Einstellung) trifft überhaupt nicht zu“ bis $5=$ „trifft voll und ganz zu“

Die Glaubwürdigkeitseinschätzung von Corporate Blogs hängt erkennbar, jedoch nicht sehr stark mit der globalen Einstellung gegenüber Unternehmen zusammen. Insbesondere die Dimensionen Kompetenz, Dynamik und Ähnlichkeit sind in dieser Hinsicht betroffen, so dass die Glaubwürdigkeit von Corporate Blogs in spezifischer Weise von allgemeiner Unternehmens-Skepsis tangiert wird. Ein Vergleich der Blogleser mit den Blogautoren in der Stichprobe zeigt, dass die negative Haltung gegenüber Unternehmen unter den aktiv schreibenden Bloggern stärker ausgeprägt ist als bei den Lesern. Die zuvor ermittelten Unterschiede in den Glaubwürdigkeitszuschreibungen zwischen Lesern und Verfassern von privaten Weblogs dürften daher zumindest teilweise dadurch begründet sein, dass die befragten Blogautoren Wirtschaftsunternehmen grundsätzlich kritischer gegenüberstehen.

\section{Diskussion}

Die Erhebung von Glaubwürdigkeitsurteilen über Corporate Blogs und private Weblogs bei Mitgliedern der deutschsprachigen Blogger-Community hat ein Glaubwürdigkeitsdefizit von Corporate Blogs zu Tage gefördert. Zugleich hat sich der konzeptuelle Zugang, Glaubwürdigkeit mehrdimensional zu messen, bewährt, weil so die Qualität der Glaubwürdigkeitsunterschiede genauer ermittelt werden kann. Demnach sehen die befragten Blogger die größten Unterschiede zwischen Corporate und privaten Blogs bei den Dimensionen Sympathie, Ähnlichkeit und soziale Billigung. Die deutlich besseren Urteile über private Blogs auf diesen Dimensionen zeigen eine affektiv aufgeladene negative Einstellung zu Corporate Blogs, die darin begründet sein könnte, dass die BloggerCommunity diesen Kommunikationskanal als egalitäres, gewissermaßen basisdemokratisches Medium begreift (vgl. z. B. Schmidt 2006), das sie nicht von Unternehmen instrumentalisiert und damit seinem Bestimmungszweck entfremdet sehen möchte. Die negativen Urteile bezüglich Sympathie und Ähnlichkeit verweisen auf diese Einschätzung als eine Art Fremdkörper in der Blogosphäre; sie drücken eine Abgrenzung ge- 
genüber Corporate Blogs im Sinne nicht übereinstimmender Wert- und Zielvorstellungen aus. Auch die geringe wahrgenommene soziale Billigung von Corporate Blogs reflektiert Einschränkungen der wahrgenommenen Kompatibilität zwischen Blogosphäre und Unternehmenskommunikation. So fügt sich auch die Erkenntnis, dass die kritische Haltung gegenüber Unternehmen die Glaubwürdigkeitsurteile über Corporate Blogs negativ beeinflusst, zu einem Gesamtbild einer PR-skeptischen Blogosphäre.

Dabei sind die Blogger durchaus bereit, die Professionalität von Corporate Weblogs anzuerkennen: Auf der Kompetenzdimension stellt sich der Unterschied zu privaten Blogs zwar immer noch deutlich, aber längst nicht so substanziell dar wie bei den zuvor angesprochenen Dimensionen. Hinsichtlich der Dimension Dynamik ergibt sich kein Unterschied. Aus der Sicht der Zielgruppe von Corporate Blogs, nämlich den Mitgliedern der Blogosphäre, erscheint die Beteiligung von Unternehmen am großen Blog-Netz daher als nicht unbedingt willkommen, wenngleich durchaus professionell umgesetzt.

Die skeptische Grundhaltung gegenüber Corporate Blogs trifft auf solche Befragte, die selbst aktiv Blogs verfassen, in geringfügig stärkerem Umfang zu als auf reine Blogleser. Dieser Unterschied steht in Verbindung mit einer bei Blogautoren stärker ausgeprägten kritischen Haltung gegenüber Wirtschaftsunternehmen. Auch wenn die Unterschiede zwischen Autoren und Lesern von Blogs nicht substanziell sind, verweist dieser Befund darauf, dass Online-Kommunikation die noch relativ kleine Gruppe der Blogautoren (vgl. Busemann/Gscheidle 2009) und potenziellen Motoren der Informationsdiffusion in der Blogosphäre besonders berücksichtigen muss. Soweit diese unternehmenskritische Haltung auf alle aktiven Blogger generalisierbar ist, ist davon auszugehen, dass positive Unternehmensmeldungen eine geringere Chance haben dürften, als Unternehmens-Kritik in der Blogosphäre Multiplikatoren zu finden. Diese Überlegungen müssten freilich durch Diffusions- und Zitationsanalysen in der Blogosphäre gegengeprüft werden. Der Befund impliziert indes nicht, dass die Erreichung spezifischer Teilpublika, etwa Journalisten, durch Corporate Blogs nicht dennoch besonders erfolgreich gelingen könnte.

Aus anwendungswissenschaftlicher Perspektive ergibt sich aus den referierten Befunden die Handlungsempfehlung an die Praxis der Online-Kommunikation, das Medium Weblog in einer Art und Weise auszugestalten, die den Erwartungen der BloggerCommunity gerecht wird und die Skepsis, die einem Corporate Blog offenbar entgegenschlägt, aktiv aufzugreifen. Falsche Darstellungen von unternehmensbezogenen Sachverhalten, Mangel an Selbstkritik, offensiv formulierte Vorwürfe an kritische private Blogger oder einseitige Blogeinträge angesichts kritischer Medienberichte oder öffentlicher Debatten bergen das Risiko, die Glaubwürdigkeitsschwächen hinsichtlich der vermuteten Ähnlichkeit von Haltungen, zugeschriebener sozialer Akzeptanz und Sympathie zu verstärken und riskieren ggf. auch eine kontraproduktive Verbreitung in der kritischen Blogosphäre.

Die Befunde erlauben aber neben Warnungen vor dem, was Online-PR vermeiden sollte, auch positive Handlungsempfehlungen. Corporate Blogger sollten sich der in der vorliegenden Studie aufgedeckten dimensionsspezifischen Glaubwürdigkeitsdefizite annehmen und insbesondere versuchen, die Werthaltungen der Blogger-Community (vgl. dazu Cenite et al. 2009) anzusprechen, damit Unternehmenskommunikation weniger als Fremdkörper und mehr als legitime, relevante Quelle aufgefasst wird. Aus dieser Sicht ist Corporate Blogging eine Plattform für die Kommunikation von Corporate Citizenship oder Corporate Social Responsibility (Esrock/Leichty 1998) und weniger für die Produkt-PR. Corporate Blogs, die der Blogosphäre das Engagement für gesellschaftliche Belange signalisieren, dürften bessere Chancen haben, die ermittelten Sympathie-, 
Ähnlichkeits-, Vertrauenswürdigkeits- und Billigungsdefizite abzubauen als marketingbeherrschte, produkt- und lobbyfokussierte Bloginhalte. Die bereits hochbewertete Professionalität bestehender Corporate Weblogs stellt einen guten Ausgangspunkt für solche Ansätze dar. Mithin kann die praktische Online-PR auch auf Gewöhnungseffekte hoffen: Die in der vorliegenden Studie beobachtete „Fremdheit“ von Corporate Blogs mag mit der Zeit abnehmen, wenn das Internet-Publikum mit der Existenz und Arbeitsweise dieses Kanals besser vertraut ist.

Für die künftige Forschung ergibt sich die Herausforderung, die Glaubwürdigkeit verschiedener Unterarten von Corporate Blogs zu untersuchen, die sich in ihren Urhebern, kommunikativen Zielen, Inhalten und Zielgruppen unterscheiden (z. B. Service Blogs versus $\mathrm{CEO} \mathrm{Blogs,} \mathrm{Blogs} \mathrm{von} \mathrm{Unternehmen} \mathrm{verschiedener} \mathrm{Größe} \mathrm{und} \mathrm{Markt-}$ macht, etwa Großkonzerne, Kleinunternehmen oder Freiberufler). Weiterhin stellt sich die Frage nach der Verflechtung von korporativer Blogkommunikation in der Blogosphäre anhand realer Botschaften sowie deren Zitation und Kommentierung durch private Blogger. Außerdem bieten sich Möglichkeiten für weitere Umfragestudien. Zwar operierte die Rekrutierungsstrategie der vorliegenden Studie über das Interesse an Blogs und dürfte daher zu einem durchaus aussagekräftigen, „typischen“ Blogger-Sample geführt haben; dennoch verbietet sich eine offensive Generalisierung der Befunde auf die gesamte Blog-Community. Mit diesen Ansätzen könnten die methodischen Limitationen der vorliegenden Studie ausgeglichen werden, die zum einen nur grundsätzliche Einstellungen zu Corporate Blogs ermitteln konnte (und damit konkretes BloggingVerhalten in Bezug auf unterschiedliche Formen von Corporate Blogs nicht abbildet) und zum anderen ein nicht repräsentatives Sample der deutschsprachigen Blogverfasser und -leser zugrundelegt.

Schließlich ist mit Blick auf die Gesamtstrategie der Unternehmenskommunikation zu untersuchen, inwiefern das Einzelinstrument Corporate Weblog ein spezifisches Glaubwürdigkeitsdefizit aufweist, das andere Instrumente (z. B. herkömmliche Websites oder eventbasierte Kundenkommunikation) nicht oder in nur geringem Umfang besitzen. Möglicherweise spiegeln die vorliegenden Befunde nur das generelle Misstrauen, das Privatbürger allen unternehmerischen Medien und Botschaften gegenüber empfinden (Callison 2001) und weniger ein blog-spezifisches Phänomen. Solche perspektivischen Studien würden es auch erlauben, den Glaubwürdigkeitsaspekt innerhalb der strategischen Instrumentenkombination eines Unternehmens angemessener zu berücksichtigen.

\section{Literatur}

Austin, E. W./Dong, Q. (1994): Source v. Content Effects on Judgments of News Believability. In: Journalism Quarterly, 71, Heft 4, S. 973-983.

Bentele, G. (1988): Der Faktor Glaubwürdigkeit. In: Publizistik, 33, Heft 2/3, S. 406-426.

Bentele, G./Seidenglanz, R. (2005): Vertrauen und Glaubwürdigkeit. In: Bentele, G./Fröhlich, R./ Szyszka, P. (Hrsg.): Handbuch der Public Relations (S. 346-360). Wiesbaden: Verlag für Sozialwissenschaften.

Berlo, D. K./Lemert, J. B./Mertz, R. J. (1969): Dimensions for Evaluating the Acceptability of Message Sources. In: Public Opinion Quarterly, 33, Heft 4, S. 563-576.

Busemann, K /Gscheidle, M. (2009): Web 2.0: Communitys bei jungen Nutzern beliebt. In: Media Perspektiven, o. Jg., Heft 7, S. 356-363.

Byrne, D. (1961): Interpersonal Attraction and Attitude Similarity. In: Journal of Abnormal Social Psychology, 62, Heft 3, S. 713-715. 
Callison, C. (2001): Do PR Practitioners Have a PR Problem? The Effect of Associating a Source with Public Relations and Client-Negative News on Audience Perception of Credibility. In: Journal of Public Relations Research, 13, Heft 3, S. 219-234.

Cenite, M./Detenber, B. H./Koh, A. W. K./Lim, A. L. H./Soon, N. E. (2009): Doing the Right Thing Online: A Survey of Bloggers' Ethical Beliefs and Practices. In: New Media \& Society, 11, Heft 4, S. 575-597.

Copeland, H. (2004): Blogads: Reader Survey for Blog Advertising. Verfügbar unter: http:// www.blogads.com/survey/blog_reader_survey.html [31.08.2007].

Deisenberg, A. M. (2007): FOCUS-Studie: 2,5 Millionen Deutsche besuchen Weblogs. Verfügbar unter: www.burda.de/hps/client/hbmi/bdspress/release_actual_long/hbmi_article_mainnav/ presse/archiv/BDSPRESS_RELEASE_3256.hbs [02.09.2009].

Eck, K. (2007): Mehr als 600 Corporate Blogs. Verfügbar unter: http://klauseck.typepad.com/ prblogger/2007/05/corporateblogs.html [02.09.2009].

Esrock, S. L./Leichty, G. B. (1998): Social Responsibility and Corporate Web Pages: Self-presentation or Agenda-setting? In: Public Relations Review, 24, Heft 3, S. 305-319.

Fischer, T. (2006): Die Entwicklung von Weblog Issues am Beispiel des Klingeltonanbieters Jamba. In: Picot, A./Fischer, T. (Hrsg.): Weblogs professionell (S. 247-252). Heidelberg: dpunkt.verlag.

Forgas, J. P. (1987): Sozialpsychologie. München-Weinheim: Psychologie Verlags Union.

Gaziano, C./McGrath, K. (1986): Measuring the Concept of Credibility. In: Journalism Quarterly, 63, Heft 3, S. 451-462.

Giffin, K. (1967): The Contribution of Studies of Source Credibility to a Theory of Interpersonal Trust in the Communication Process. In: Psychological Bulletin, 68, Heft 2, S. 104-120.

Horowitz, A. (2005, 1. Januar): 101 Dumbest Moments in Business. Verfügbar unter: http://money.cnn.com/magazines/business2/business2_archive/2005/01/01/8250237/index.htm [02.09.2009].

Hovland, C. I./Weiss, W. (1951): The Influence of Source Credibility on Communication Effectiveness. In: Public Opinion Quarterly, 15, Heft 4, S. 635-650.

Hubig, C./Siemoneit, O. (2007). Vertrauen und Glaubwürdigkeit in der Unternehmenskommunikation. In: Piwinger, M. (Hrsg.): Handbuch Unternehmenskommunikation (S. 171-189). Wiesbaden: Gabler.

Jacobson, H. K. (1969): Mass Media Believability: A Study of Receiver Judgements. In: Journalism Quarterly, 46, Heft 1, S. 20-28.

Johnson, T. J./Kaye, B. K. (2004): Wag the Blog: How Ralience on Tradional Media and the Internet Influence Credibility Perceptions of Weblogs among Blog Users. In: Journalism and Mass Communication Quaterly, 81, Heft 3, S. 622-642.

Kelleher, T./Miller, B. M. (2006): Organizational Blogs and the Human Voice: Relational Strategies and Relational Outcomes. In: Journal of Computer-Mediated Communication, 11(2), Artikel 1. http://jcmc.indiana.edu/vol11/issue2/kelleher.html [02.09.2009].

Köhnken, G. (1990): Glaubwürdigkeit. Untersuchungen zu einem psychologischen Konstrukt. München: Psychologie Verlags Union.

Lange, M. (2006): Corporate Blogs stärken Glaubwürdigkeit von Unternehmen. Verfügbar unter www.pr-guide.de/index.php?id=192\&tx_ttnews[tt_news]=995\&tx_ttnews[backPid $]=251 \& \mathrm{cHash}=8 \mathrm{bbc} 834766$ [02.09.2009].

Lowry, D. T. (1973): Demographic Similarity, Attitudinal Similarity, and Attitude Change. In: Public Opinion Quarterly, 37, Heft 2, S. 192-208.

Mast, C. (2006): Unternehmenskommunikation (2. Aufl.). Stuttgart: Lucius \& Lucius.

Matthes, J./Kohring, M. (2003): Operationalisierung von Vertrauen in Journalismus. In: Medien \& Kommunikationswissenschaft, 51, Heft 1, S. 5-23.

McCroskey, J. C./Jenson, T. A. (1975): Image of Mass Media News Sources. In: Journal of Broadcasting, 19, Heft 2, S. 169-180.

Meyer, P. (1988): Defining and Measuring Credibility of Newspapers: Developing an Index. In: Journalism Quarterly, 65, Heft 2, S. 567-574.

Mosier, N. R./Ahlgren, A. (1981): Credibility of Precision Journalism. In: Journalism Quarterly, 58, Heft 3, S. 375-381. 
Nawratil, U. (1997): Glaubwürdigkeit in der sozialen Kommunikation. Opladen: Westdeutscher Verlag.

Nawratil, U. (1999): Glaubwürdigkeit als ein Faktor im Prozess medialer Kommunikation. In: Rössler, P./Wirth, W. (Hrsg.): Glaubwürdigkeit im Internet. Fragestellungen, Modelle, empirische Befunde (S. 15-31). München: Verlag Reinhard Fischer.

Neuberger, C. (2005): Formate der aktuellen Internetöffentlichkeit. In: Medien \& Kommunikationswissenschaft, 53, Heft 1, S. 73-92.

Neuberger, C., Nuernbergk, C./Rischke, M. (2007): Weblogs und Journalismus: Konkurrenz, Ergänzung oder Integration? In: Media Perspektiven, o. Jg., Heft 2, S. 96-112.

Noelle-Neumann, E./Köcher, R. (Hrsg.) (1997): Allensbacher Jahrbuch der Demoskopie 1993-1997 (Band 10). München: Saur.

Pelz, J./Scholl, W. (1990): Entwicklung eines Verfahrens zur Messung von Sympathie, Einwirkung, Macht-Einfluss-Differenzierung und Interesse (SEMI). Göttingen: Georg-August-Universität, Institut für Wirtschafts- und Sozialpsychologie (IWSP-Bericht 17).

Picot, A./Fischer, T. (Hrsg.) (2006): Weblogs professionell. Heidelberg: dpunkt.verlag.

Pleil, T. (2004): Meinung machen im Internet? Personal Web Publishing und Online-PR. Verfügbar unter: www.pr-guide.de/index.php?id=192\&tx_ttnews[tt_news]=304 [02.09.2009].

Röttger, U./Zielmann, S. (2006): Weblogs - unentbehrlich oder überschätzt für Kommunikationsmanagement von Organisationen? In: Picot, A./Fischer, T. (Hrsg.): Weblogs professionell (S. 31-50). Heidelberg: dpunkt.verlag.

Schmidt, J. (2006): Weblogs: Eine kommunikationssoziologische Studie. Konstanz: UVK.

Schmidt, J./Frees, B./Fisch, M. (2009): Themenscan im Web 2.0. In: Media Perspektiven, o. Jg., Heft 2, S. 50-59.

Scholz, J. (2005): Brandbriefe aus dem Netz. In: Werben \& Verkaufen, o. Jg., Heft 5, S. 34.

Schwarzer, P./Sarstedt, M./Baumgartner, A. (2007): Corporate Blogs als Marketinginstrument. Saarbrücken: VDM.

Schweiger, W. (1998): Wer glaubt dem World Wide Web? Ein Experiment zur Glaubwürdigkeit von Nachrichten in Tageszeitungen und im World Wide Web. In: Rössler, P. (Hrsg.): OnlineKommunikation. Beiträge zu Nutzung und Wirkung (S. 123-145). Wiesbaden: Westdeutscher Verlag.

Simons, H. W./Berkowitz, N. N./Moyer, R. J. (1970): Similarity, Credibility, and Attitude Change: A Review and a Theory. In: Psychological Bulletin, 73, Heft 1, S. 1-16.

Singletary, M. W. (1976): Components of Credibility of a Favourable News Source. In: Journalism Quarterly, 53, Heft 2, S. 316-319.

Sonnabend, L. (2005): Das Phänomen Weblogs - Beginn einer Medienrevolution? Magisterarbeit am Institut für Kommunikationswissenschaft und Medienforschung der Ludwig-MaximiliansUniversität München. Verfügbar unter: www.netzthemen.de/sonnabend-weblogs [02.09.2009].

Thimm, C./Berlinecke, A. (2007): Mehr Öffentlichkeit für unterdrückte Themen? Chancen und Grenzen von Weblogs. In: Pöttker, H./Schulzki-Haddouti, C. (Hrsg.): Vergessen? Verschwiegen? Verdrängt? Zehn Jahre „Initiative Nachrichtenaufklärung“ (S. 81-101). Wiesbaden: VS Verlag.

Wheeless, L. R. (1976): Self-Disclosure and Interpersonal Solidarity: Measurement, Validation, and Relationship. In: Human Communication Research, 3, Heft 1, 47-61.

White, C./Raman, N. (1999): The World Wide Web as a Public Relations Medium: the Use of Research, Planning, and Evaluation in Web Site Development. In: Public Relations Review, 25, Heft 4, S. 405-419.

Wirth, W. (1999): Methodologische und konzeptionelle Aspekte der Glaubwürdigkeitsforschung. In: Rössler, P./Wirth, W. (Hrsg.): Glaubwürdigkeit im Internet. Fragestellungen, Modelle, empirische Befunde (S. 47-66). München: Verlag Reinhard Fischer.

Wolff, P. (2006): Die Macht der Blogs. Chancen und Risiken von Corporate Blogs und Podcasting in Unternehmen. Frechen: Datakontext.

Yang, S.-U./Lim, J. S. (2009): The Effects of Blog-Mediated Public Relations (BMPR) on Relational Trust. In: Journal of Public Relations Research, 21, Heft 3, S. 341-359.

Zerfaß, A. (2004): Unternehmensführung und Öffentlichkeitsarbeit (2. Aufl.). Wiesbaden: VS Verlag. 
Zerfaß, A. (2005a): Corporate Blogs: Einsatzmöglichkeiten und Herausforderungen [Elektronische Version]. Verfügbar unter: www.zerfass.de/CorporateBlogs-AZ-270105.pdf [02.09.2009].

Zerfaß, A. (2005b): Weblogs als Meinungsmacher. Neue Spielregeln für die Unternehmenskommunikation. Verfügbar unter: www.hlv-kommunikationsmanagement.de. [02.09.2009].

Zerfaß, A./Bogosyan, J. (2007): Blogstudie 2007. Informationssuche im Internet - Blogs als neues Recherchetool. Ergebnisbericht der Universität Leipzig. Verfügbar unter: http://www.blogstudie2007.de/inc/blogstudie2007_ergebnisbericht.pdf [02.09.2009].

Zerfaß, A./Sandhu, S. (2006): CEO-Blogs: Personalisierung der Online-Kommunikation als Herausforderung für die Unternehmensführung. In: Picot, A./Fischer, T. (Hrsg.): Weblogs professionell (S. 51-66). Heidelberg: dpunkt.verlag.

\section{Filmfinanzierung im Vergleich}

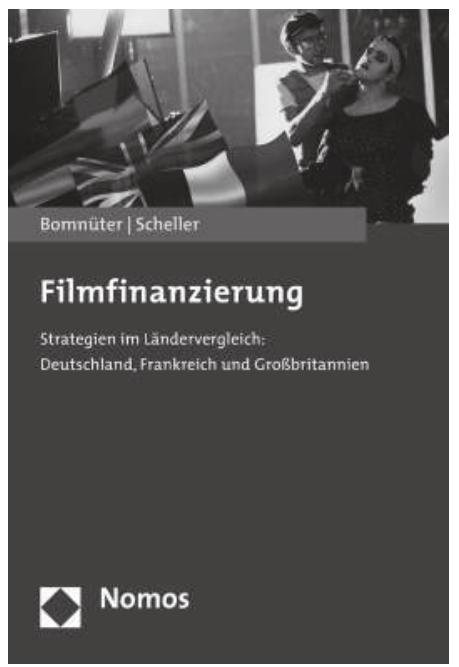

\section{Filmfinanzierung}

Strategien im Ländervergleich:

Deutschland, Frankreich und

Großbritannien

Von Udo Bomnüter

und Patricia Scheller

2009, 123 S., brosch., 19,- $€$,

ISBN 978-3-8329-4908-2

Europäische Kinofilmproduzenten haben die unterschiedlichsten Finanzierungsstrategien entwickelt, um sich gegen die erdrückende Konkurrenz der US-Majors zu behaupten. Allerdings fand bislang kein Vergleich landesspezifischer Ansätze statt. Diese Lücke soll das vorliegende Buch schließen. Erstmalig werden Finanzierungsbausteine in Deutschland, Frankreich und Großbritannien aus einer komparativen Perspektive analysiert. 